\title{
CIENCIA\&SALUD
}

\section{Diagnóstico y manejo de retinoblastoma en pediatría.}

\section{Diagnosis and management of retinoblastoma in pediatrics.}

Juan Diego Vargas Cedeño ${ }^{1}$, Catalina Castrillo Hine², Leslie Argüello Cruz³

1 Médico residente, Caja costarricense de seguro social, San José Costa Rica.

2 Bachiller en ciencias de la salud. Trabajador independiente, San José Costa Rica

3 Médico oftalmólogo pediatra. Caja costarricense de seguro social, San José Costa Rica.

Contactos:juandivc@hotmail.com catalina.castrillohine@gmail.com larguelloc@ccss.sa.cr

\section{RESUMEN}

\section{Cómo citar:}

Vargas Cedeño, J.

D., Castrillo Hine,

C., \& Argüello Cruz,

L. Perlas sobre

detección y manejo

de Retinoblastoma en

Pediatría. Revista Ciencia

Y Salud Integrando

Conocimientos, 5(5),

Pág. 113-122. https://

doi.org/10.34192/

cienciaysalud.v5i5.369

Recibido: $02 / \mathrm{jul} / 2021$

Aceptado: 12 /oct/2021

Publicado: $18 /$ oct/2021
El retinoblastoma es un cáncer que surge debido a que se pierden ambas copias del gen RB1 que normalmente tienen como función suprimir la aparición de esta neoplasia en una célula retiniana en desarrollo. El retinoblastoma puede afectar uno o ambos ojos y se ha documentado que hasta el $5 \%$ de los niños con retinoblastoma de tipo hereditario asocia concomitantemente un tumor cerebral de línea media. Sin un tratamiento oportuno y eficaz, esta enfermedad puede diseminarse a través del nervio óptico o por vía hematógena, causando diversas manifestaciones y complicaciones que incluso pueden provocar la muerte. Actualmente, se desconoce el mecanismo específico por el cual se ocasiona el daño genómico del gen RB1. A pesar de esto, el retinoblastoma se desarrolla como una patología relativamente común en todas las etnias, independientemente del entorno geográfico y estrato social, por lo que su diagnóstico y manejo temprano implican una responsabilidad médica general.

Palabras Clave: Retinoblastoma, neoplasia, retinopatía del prematuro, tratamiento.

\section{ABSTRACT}

Retinoblastoma is a type of cancer that arises due to the loss of both copies of the RB1 gene that normally has the function of suppressing the appearance of this neoplasm in a developing retinal cell. Retinoblastoma can affect one or both eyes, and it has been documented that up to $5 \%$ of the children with hereditary retinoblastoma can also present a midline brain tumor. Without prompt and effective treatment, this disease can spread through the optic nerve or the hematogenous pathway, causing multiple manifestations and complications that can even lead up to death. Currently, the specific mechanism by which the genomic damage of the RB1 gene is caused is still unknown. Regardless of this, retinoblastoma develops as a relatively common pathology in all ethnic groups, in spite of the geographic environment and the social stratum, therefore its early diagnosis and management implies a general medical responsibility among all physicians.

Keywords: Retinoblastoma, neoplasia, retinopathy of prematurity, treatment. 


\section{INTRODUCCIÓN}

El retinoblastoma es la neoplasia maligna intraocular primaria más común de la infancia y representa del 10-15\% de los cánceres que ocurren durante el primer año de vida, afectando de 1:15 000 a 1:18 000 nacidos vivos (1,2). Este se presenta de forma hereditaria y no hereditaria $(1,3)$.

La mediana de edad al momento del diagnóstico es de 18-20 meses, con un promedio de 12 meses para niños con enfermedad bilateral y 24 meses para niños con enfermedad unilateral (4). La incidencia es similar entre niños y niñas, y no hay predilección racial (5). En general, el 95\% de los niños con retinoblastoma se presenta antes de los cinco años.

La única forma de diagnosticar un retinoblastoma de manera temprana es bajo la sospecha clínica y un examen físico realizado bajo el entrenamiento necesario y especializado. Sin embargo, si se tiene la sospecha y se examina tempranamente la retina de pacientes con historia familiar positiva, se pueden detectar lesiones altamente sugestivas del tumor con lo que se pueden prevenir muertes y complicaciones graves. Esta debería ser la principal prioridad del tratamiento, seguido de recuperación de la visión y de ser posible prevenir comorbilidades y neoplasias secundarias.

\section{MÉTODOS}

Se realizó una revisión bibliográfica incluyendo metaanálisis, reportes de caso, reportes de bases de datos estadísticos, artículos originales y artículos de revisión de la base de datos PubMed.

\section{Predisposición genética}

Los niños con antecedentes familiares de retinoblastoma tienen un riesgo elevado de padecerlo y requieren de mayor vigilancia para detectar el desarrollo de tumores retinianos (6). Sin embargo, estos pacientes representan menos del 10\%, lo que sugiere que la mayoría de los casos surge de mutaciones somáticas o mutaciones de novo en la línea germinal (6). Las mutaciones de la línea germinal en el gen del retinoblastoma (RB1) están presentes en el $40 \%$ de los casos, predominantemente en la enfermedad bilateral. Por otro lado, en el retinoblastoma no hereditario surgen nuevas mutaciones somáticas en la célula retiniana.

Existe una estimación del riesgo de desarrollar retinoblastoma según la relación del paciente con el miembro de la familia que porte el antecedente y este puede definirse con mayor precisión mediante el análisis genético familiar. Esto consta de la realización de pruebas genéticas del gen RB1 de un familiar afectado para identificar la heredabilidad. Dichas pruebas permiten identificar a los pacientes con alto riesgo de presentar la enfermedad, a quienes se les debe de dar un seguimiento más detallado. Cabe destacar que la mayoría de familiares considerados de riesgo que no portan la mutación no requieren otras pruebas $(7,8)$.

Las pruebas genéticas del paciente o familiar afectado también permiten determinar el riesgo de tumores adicionales y segundas neoplasias malignas primarias para las cuales las personas con mutaciones de la línea germinal RB1 tienen un potencial riesgo elevado $(7,9)$. La anomalía genética asociada con el desarrollo de retinoblastoma hereditario también se asocia a un mayor riesgo de desarrollar segundas neoplasias malignas, como el sarcoma osteogénico, sarcomas de tejidos blandos, en particular el leiomiosarcoma y melanoma maligno (10).

El 7-10\% de los retinoblastomas son neonatales y se diagnostican durante el primer mes de vida, ocasionalmente al nacer (11). Existen pruebas como la amniocentesis usada como cribado prenatal en parejas con una mutación conocida de RB1. En general, se diagnostica en menores de 3 años, siendo poco común el diagnóstico después de los 8 años (12). No se documentan diferencias consistentes en la incidencia asociadas a algún origen étnico o geográfico. Sin embargo, recientemente se informaron disparidades raciales y étnicas en los Estados Unidos, con una incidencia significativamente mayor de retinoblastoma bilateral en la población hispana (13). Además, existen datos que sugieren la asociación de la edad paterna 


\section{CIENCIA\&SALUD}

con riesgo de retinoblastoma esporádico, donde el riesgo aumenta según la edad, especialmente la materna. De igual manera existe literatura que asocia al tabaquismo activo materno durante el embarazo (12).

\section{Patogénesis}

El comportamiento biológico y clínico del retinoblastoma está dictado por las características únicas de las células oncogénicas y sus interacciones con el huésped. Estas son determinadas por la expresión génica intrínseca junto con cambios en la señalización de la célula de origen inducidos por mutaciones oncogénicas. A diferencia de la mayoría de neoplasias, los cambios genéticos que inician el retinoblastoma están bien definidos y sus mecanismos están en estudio (14).

El retinoblastoma generalmente es causado por la inactivación de ambos alelos del gen del RB1 (1). Este gen pertenece al cromosoma 13q14 y codifica una proteína nuclear supresora de tumores. Esta proteína restringe la capacidad de la célula para progresar de fase $\mathrm{G} 1$ a fase $\mathrm{S}$ del ciclo celular, por lo que su pérdida, provoca una desregulación del ciclo celular $(15,16)$.

El retinoblastoma hereditario tiene riesgo de tumores multifocales y bilaterales, este muestra una penetrancia incompleta, donde el $90 \%$ de los portadores genotípicos expresan la malignidad. El mosaicismo por una mutación en el gen RB1 también puede causar enfermedad (15). En la mayoría de los no hereditarios, ambas mutaciones surgen espontáneamente en una célula somática de la retina, lo cual resulta en el escenario habitual de tumor unilateral unifocal $(15,16)$.

El retinoma ha surgido como intermediario en la génesis del retinoblastoma. En un estudio, el $20 \%$ de los pacientes enucleados con retinoblastoma tenían un componente de retinoma ubicado hacia la base. Se presume que la transformación de retinomas de larga duración es el responsable de la aparición de retinoblastoma del adulto (17).

\section{Historia natural de la enfermedad}

El retinoblastoma sin tratamiento es una enfermedad mortal ya que estos tumores infiltran el globo ocular y lo destruyen. El pronóstico de rescate ocular es bajo y depende del estadio al momento del diagnóstico. Además, la diseminación metastásica se detecta dentro de los primeros 12 meses de la presentación clínica. La regresión espontánea puede ocurrir, pero es rara (18). La presentación de un retinoblastoma metastásico al diagnóstico es poco común en países de primer mundo, pero es la causa más frecuente de muerte por este tumor a nivel mundial $(19,20)$.

La mediana de tiempo entre el diagnóstico intraocular principalmente enucleado con factores de riesgo patológico alto y la primera evidencia de metástasis es de 10 meses en pacientes con quimioterapia adyuvante, pero es menos de 5 meses si no reciben tratamiento (19). Las vías más comunes de diseminación metastásica son la infiltración directa por el nervio óptico hacia el sistema nervioso central o a través de la coroides hacia la esclerótica y la órbita $(18,19,20)$.

\section{DIAGNÓSTICO}

Es el único cáncer pediátrico que presenta signos reconocibles y que no requiere de estudios patológicos para confirmar su diagnóstico debido a sus características patognomónicas funduscópicas. Esta característica única del confiere el potencial de mejorar la supervivencia y la integridad del globo ocular con la detección temprana.

La presentación clásica temprana es una masa intrarretiniana translúcida, solitaria o multifocal, bien circunscrita. A medida que avanza, el tumor se vuelve rosado, con vasos sanguíneos dilatados y puede exhibir uno de los tres patrones de crecimiento principales.

Se presenta generalmente como leucocoria en menores de 2 años. La leucocoria, un reflejo pupilar blanquecino, es el signo característico en el $60 \%$ de los casos. Otros signos y síntomas incluyen el 


\section{CIENCIA\&SALUD}

estrabismo, nistagmo y el ojo rojo. La edad promedio al diagnóstico es 20 meses. Actualmente, se ha logrado identificar la leucocoria al realizar fotografías con flash, hallazgo que precede al diagnóstico por varios meses, especialmente cuando el modo anti-reflejo de ojos rojos de la cámara está inactivado, evitando la constricción de la pupila por el flash $(3,21)$.

El estrabismo ocurre con la pérdida de visión central por afectación macular. Es el segundo signo más frecuente, presente en el $20 \%$ y se detecta más temprano con enfermedad menos avanzada que la leucocoria (4). Otras manifestaciones menos comunes son la disminución de la agudeza visual (4\%), inflamación ocular (5\%), heterocromía del iris, hemorragia vítrea, hipema, glaucoma, anisocoria y celulitis orbitaria (22). La enfermedad extraocular es más común al diagnóstico en países en desarrollo. Los síntomas en enfermedad metastásica pueden incluir anorexia, pérdida de peso, vómitos, cefalea, masa orbitaria, deterioro neurológico o masa de tejidos blandos (23).

La evaluación inicial incluye un examen físico completo, examen oftalmológico bajo anestesia, ecografía ocular, tomografía de coherencia óptica y resonancia magnética de cerebro y órbitas $(24,25,26,27,28)$. La oftalmoscopia indirecta bajo anestesia es el estándar de oro para la detección del retinoblastoma y suele ser suficiente para establecer el diagnóstico. El uso de fotografía del fondo de ojo permite monitorizar la terapia en el manejo conservador ya que brinda una representación y comparación más objetiva de las imágenes $(25,27)$.

La ecografía es sugestiva si existe calcificación dentro de la masa y el globo ocular es de tamaño normal (27). Las imágenes de tomografía de coherencia óptica pueden detectar pequeños tumores no visibles en la funduscopía y se utiliza para vigilancia durante y después del tratamiento para detectar recurrencias enmascaradas por cicatrices retinianas (28).

La resonancia magnética nuclear evalúa el tamaño del tumor, la afectación del nervio óptico y la presencia de un tumor intracraneal (26). Es la técnica más sensible para evaluar extensión extraocular. Por otro lado, la angiografía con fluorosceina del segmento anterior y posterior juega un papel fundamental en la evaluación en el contexto de quimioterapia intraarterial al documentar actividad tumoral o complicaciones vasculares, para detectar neovascularización subclínica del iris y evaluar la vascularización tumoral y retiniana (29).

La biomicroscopía ultrasónica produce imágenes de alta resolución del segmento anterior y estructuras de la cámara posterior ocultas, como el cuerpo ciliar, las zónulas o la hialoides anterior. Durante la última década, ha demostrado ser fundamental en la evaluación de extensión anterior, donde la invasión tumoral de la cámara posterior se puede detectar con alta sensibilidad (81\%) y especificidad (100\%), incluso en ausencia de afectación de la cámara anterior (30). En la práctica clínica funciona para evaluar la extensión anterior al momento del diagnóstico o la integridad de la hialoides anterior y la úvea anterior en la recaída.

\section{Pruebas genéticas}

Se sugieren pruebas genéticas moleculares a todos los pacientes afectados ya que las formas hereditarias, las pruebas de leucocitos pueden identificar la mutación en el 90-95\% (7). Estas permiten estimar el riesgo de enfermedad en la familia y la futura descendencia, evitando un cribado clínico innecesario a los hermanos del paciente (28).

Las pruebas moleculares deben realizarse inicialmente en células tumorales de estar disponibles en pacientes con enucleación para poder identificar la mutación específica.

El diagnóstico prenatal se recomienda para niños con antecedentes familiares positivos. Actualmente, la extracción de ADN para el análisis mutacional puede adquirirse con el muestreo de vellosidades coriónicas al final del primer trimestre o con la amniocentesis durante el segundo trimestre (31). 


\section{CIENCIA\&SALUD}

\section{Retinoblastoma trilateral}

Al sufrir una transformación maligna, el retinoblasto produce un tumor intrarretiniano primario con capacidad de metástasis y de generar otros tumores. El retinoblastoma trilateral consiste en un retinoblastoma uni o bilateral asociado a un tumor intracraneal similar histológicamente (32).

El 75\% surge en la glándula pineal, pero puede aparecer en las regiones supraselar o paraselar. Los tumores intracraneales ocurren en el $5 \%$ de pacientes con retinoblastoma bilateral o hereditario. Por el contrario, los tumores intracraneales son raros en los casos de retinoblastoma unilateral no hereditario y ocurren en el $0,5 \%$. En más del 50\%, el tumor intracraneal se detecta al diagnóstico y los tumores restantes ocurren después del diagnóstico, antes de los 5 años (32). En un estudio, la edad promedio al diagnóstico fue de 31 meses con un intervalo entre diagnóstico de retinoblastoma y tumor intracraneal de 21 meses.

Los estudios de patología no son necesarios para confirmar el diagnóstico. La biopsia está contraindicada por el riesgo de diseminación tumoral. Las pruebas de ADN derivado de tumores en el humor acuoso extraídas de ojos sometidos a terapia de rescate son técnicas novedosas que se han descrito como un sustituto de la biopsia de tumor $(14,25,33)$.

\section{Diagnóstico diferencial}

El diagnóstico diferencial incluye afecciones que causan leucocoria, como: persistencia hiperplasica del vítreo primario, fibrodisplasia retrolental, enfermedad de Coats, la presencia de cataratas congénitas, Toxocariasis y Toxoplasmosis ocular (24).

En una serie retrospectiva de 2775 pacientes referidos por retinoblastoma, el 22\% fueron diagnosticados con otras afecciones Estos diagnósticos varían según la edad de presentación. Los menores de 1 año se pueden presentar con vasculatura fetal persistente, enfermedad de Coats y hemorragia vítrea; los de 1-2 años con enfermedad de Coats, persistencia de la vasculatura fetal y hemorragia vítrea; los de 2-5 años con enfermedad de Coats, toxocariasis y persistencia de la vasculatura fetal; y los mayores de 5 años con enfermedad de Coats, Toxocariasis y Vitreorretinopatía exudativa familiar (34).

\section{Estrategias de tratamiento}

El objetivo principal del tratamiento es preservar la vida, con la supervivencia ocular, la preservación de la agudeza visual y la calidad de vida como objetivos secundarios (35, 36, 37). La enucleación fue el primer enfoque exitoso en disminuir la mortalidad, seguido por los intentos de rescate ocular con radioterapia. Actualmente, las opciones terapéuticas de primera línea incluyen quimioterapia local y sistémica, crioterapia, fotoablación con láser, placas radiactivas (braquiterapia con I-125) y la enucleación $(35,36,37)$.

El abordaje se basa en la clasificación internacional de retinoblastomas intra-oculares, la cual se sale del enfoque de la presente revisión. Por un lado, la mayoría de tumores extrafoveales pequeños unilaterales o bilaterales sin siembra subretiniana o vítrea se pueden tratar con técnicas focales, como: crioterapia, fotocoagulación con láser o radioterapia con placa. En tumores que involucran la mácula, la fotocoagulación con láser y la crioterapia pueden comprometer la visión central. Por lo tanto, la quimioterapia local o sistémica se utilizan para disminuir el tamaño previo a terapia focal $(35,36)$.

El tratamiento del retinoblastoma intraocular avanzado se adapta a la carga tumoral para rescatar el globo ocular (37). Los casos unilaterales del grupo C y D, se tratan con quimiocirugía de arteria oftálmica o quimioterapia IV (24). La enucleación puede ser necesaria en tumores del grupo D, en particular si el paciente es joven con enfermedad unilateral avanzada. Para los pacientes lactantes menores, la quimioterapia sistémica de agente único se puede utilizar como "terapia puente" para brindar tiempo para que el paciente crezca hasta que permita una canulación arterial, momento para realizar la quimiocirugía de la arteria oftálmica con menor riesgo $(35,37)$. 
Los pacientes con tumores unilaterales del grupo E son tratados con enucleación. Se administra quimioterapia y radioterapia adyuvantes si hay presencia de residuos microscópicos en la sección del nervio óptico o la esclerótica o si existen otros factores de riesgo patológicos $(24,37)$. Para pacientes con patología bilateral, por ejemplo los clasificados como grupo C, D, o E, las opciones de tratamiento incluyen el tratamiento focal como la crioterapia y la fotocoagulación con láser para el ojo menos afectado si el tumor es pequeño; y extrafoveal con enucleación y quimioterapia adyuvante si existen factores de riesgo patológicos. Si un ojo está principalmente enucleado, se puede utilizar quimioterapia para intentar salvar el segundo. Cuando se intentan salvar ambos ojos, se puede dar quimioterapia sistémica o quimiocirugía simultánea de la arteria oftálmica como terapia primaria.

En los casos de enfermedad trilateral, el tratamiento es la resección neuroquirúrgica seguida de quimioterapia y radioterapia craneal o craneoespinal. A pesar de la terapia agresiva, el trilateral generalmente es letal $(26,37,38)$. El uso de quimioterapia intensiva combinados con rescate de células madre hematopoyéticas autólogas se asocia con una supervivencia libre de enfermedad prolongada en series de casos $(26,37,38)$.

Los casos fallidos o recurrentes pueden tratarse con repetición de fotocoagulación con láser, crioterapia, braquiterapia con placa, quimiocirugía de la arteria oftálmica, quimiorreducción o quimioterapia intravítrea, según el tamaño, la ubicación y el historial de tratamiento previo. Para tumores de alto riesgo, se requiere de enucleación, sin embargo, muchos centros usan quimiocirugía de la arteria oftálmica con quimioterapia intravítrea para tumores del grupo D que fracasan en la terapia inicial con recuperación del globo ocular del $70 \%(39,40)$.

Los agentes que se utilizan con mayor frecuencia para la quimiocirugía de la arteria oftálmica incluyen melfalán, carboplatino y topotecán. Los agentes más usados para regímenes de terapia sistémica son carboplatino, vincristina, etopósido y topotecán $(40,41)$. En un esfuerzo por disminuir los efectos sistémicos al brindar quimioterapia en dosis altas al tumor, se instauró la quimiocirugía de la arteria oftálmica (40). Este procedimiento se realiza introduciendo una cánula a través de la arteria femoral y avanzando hasta el ostium de la arteria oftálmica, posteriormente se administra quimioterapia en la arteria oftálmica durante 30 minutos de forma pulsátil bajo anestesia general, asociado a un descongestionante nasal que se rocía en la fosa nasal del lado del tratamiento para desviar la sangre de la mucosa nasal. Esto para reducir los efectos secundarios locales. En general, se cree que en comparación con la quimioterapia sistémica, que rara vez cura el retinoblastoma intraocular por sí sola, la quimiocirugía puede alcanzar niveles más altos de fármaco en el ojo con el potencial de aumentar las tasas de curación de la enfermedad avanzada (41, 42, 43, 44).

La quimioterapia intravítrea se usa junto con la quimiocirugía de la arteria oftálmica o la quimioterapia sistémica para el tratamiento de las semillas vítreas recurrentes con una tasa de éxito cercana al 100\%. La quimioterapia sistémica se puede utilizar en: tumores más pequeños que inciden en la fóvea para encoger el tumor antes de la terapia focal, pacientes con tumores grandes, para salvar el globo ocular y encoger tumores demasiado grandes para la terapia local, bebés pequeños como "terapia puente" para proporcionar tiempo hasta que se permita la canulación arterial, tratamiento adyuvante después de la enucleación en pacientes con riesgo de metastasis y para el retinoblastoma trilateral y metastasis $(35,36,37,40,42,43)$.

La crioterapia o la fotocoagulación con láser se pueden utilizar para tratar tumores más pequeños $(<6 \mathrm{~mm}$ de diámetro y $<3 \mathrm{~mm}$ de espesor) y se pueden usar junto con la quimioterapia. Además, la terapia con placa radiactiva (braquiterapia con 1-125) consiste en asegurar una placa radiactiva en la esclerótica en la base del tumor. La dosis de radiación es de 40- 45 gray (Gy) administrada al vértice del tumor. Esta terapia logra el control del tumor en el $80 \%$ de los tumores y es más eficaz en tumores pequeños sin siembra vítrea o subretiniana.

La enucleación generalmente está indicada para el grupo E, que tiene tumores grandes con un potencial visual limitado o nulo. La enucleación se realiza si el ojo afectado tiene glaucoma secundario, mala visión o invasión de la cámara anterior (45). En estos pacientes se coloca un implante orbitario al momento de la 


\section{CIENCIA\&SALUD}

enucleación. Una vez que la conjuntiva suprayacente cicatriza, el oftalmólogo puede colocar una prótesis. Los pacientes que se someten a enucleación deben ser monitoreados para detectar posibles recaídas orbitarias, especialmente en los próximos dos años de realizada la cirugía (45).

\section{Seguimiento}

Según menciona Rodríguez-Rodríguez, et al., en su reporte de caso y revisión sobre retinoblastoma, se recomienda seguimiento cada 2-4 meses por al menos 2 años y medio hasta los 7 años de edad. Como se evidencia en dicho caso, pacientes sin manejo expedito y eficaz presentarán un desenlace progresivo asociado a complicaciones mayores $(46,20)$.

Además, se recomienda que casos con retinoblastoma germinolineal tengan control con resonancia magnética cada 3 meses en el primer año posdiagnóstico y bianual por los siguientes 3 años (46).

\section{Pronóstico y supervivencia}

El retinoblastoma no tratado es mortal, con los avances en tratamiento, la supervivencia actualmente es de más del 95\% (4). Esta varía geográficamente, desde 30\% en países en vías de desarrollo hasta 90\% países de primer mundo (47). Los pacientes con enfermedad metastásica suelen hacerla al año del diagnóstico. Un paciente libre de recurrencias por 5 años seguidos posterior al diagnóstico se considera libre de enfermedad (47).

El pronóstico de la visión en enfermedad bilateral depende del grado de afectación. El pronóstico es mejor para tumores pequeños, que no afectan la fóvea con desprendimiento y una siembra de retina limitados. Los factores que predicen la conservación de la agudeza visual de 20/40 o mejor incluyen: ubicación mayor a $3 \mathrm{~mm}$ de la fóvea y el disco óptico, y la ausencia de líquido subretiniano. Los factores que predicen mala visión en pacientes del grupo D incluyen afectación de más del 50\% de la mácula, desprendimiento de retina completo y tres o más cuadrantes de siembra de vítreo al diagnóstico. Para enfermedad unilateral, el pronóstico de la visión en el ojo sano es excelente. El desarrollo de tumores en el ojo contralateral después de transcurrir tres años es raro (47).

\section{CONCLUSIONES}

El retinoblastoma es la neoplasia maligna intraocular primaria más común de la infancia. Su diagnóstico se puede hacer con base en el examen oftalmoscópico indirecto y estudios de imagen con hallazgos particulares sin necesidad de confirmar el diagnóstico histológicamente. La referencia inmediata a un oftalmólogo pediatra y el manejo adecuado por un equipo multidisciplinario son necesarios para optimizar el resultado visual y la supervivencia del paciente.

\section{Conflictos de Interés}

La presente investigación no posee ningún conflicto de interés por declarar.

\section{Financiamiento}

La presente investigación no ha recibido financiamiento de ninguna entidad.

\section{REFERENCIAS BIBLIOGRÁFICAS}

1. Howlader N, Noone AM, Krapcho M, Garshell J, Miller D, Altekruse SF, Kosary CL, Yu M, Ruhl J, Tatalovich Z,Mariotto A, Lewis DR, Chen HS, Feuer EJ, Cronin KA (2012). SEER Cancer Statistics Review, 1975-2012. Bethesda, MD: National Cancer Institute; 2014.

2. Moll A, Kuik D, Bouter L, Den Otter W, Bezemer P, Koten J et al. Incidence and survival of 
retinoblastoma in the Netherlands: a register based study 1862-1995. British Journal of Ophthalmology. 1997;81(7):559-562.

3. Abramson D, Beaverson K, Sangani P, Vora R, Lee T, Hochberg $H$ et al. Screening for Retinoblastoma: Presenting Signs as Prognosticators of Patient and Ocular Survival. PEDIATRICS. 2003;112(6):1248-1255.

4. Abramson D, Frank C, Susman M, Whalen M, Dunkel I, Boyd N. Presenting signs of retinoblastoma. The Journal of Pediatrics. 1998;132(3):505-508.

5. Broaddus E, Topham A, Singh A. Incidence of retinoblastoma in the USA: 1975-2004. British Journal of Ophthalmology. 2008;93(1):21-23.

6. Rothschild P, Lévy D, Savignoni A, Lumbroso-Le Rouic L, Aerts I, Gauthier-Villars M et al. Familial retinoblastoma: fundus screening schedule impact and guideline proposal. A retrospective study. Eye. 2011;25(12):1555-1561.

7. Richter S, Vandezande K, Chen N, Zhang K, Sutherland J, Anderson J et al. Sensitive and Efficient Detection of RB1 Gene Mutations Enhances Care for Families with Retinoblastoma. The American Journal of Human Genetics. 2003;72(2):253-269.

8. Skalet A, Gombos D, Gallie B, Kim J, Shields C, Marr B et al. Screening Children at Risk for Retinoblastoma. Ophthalmology. 2018;125(3):453-458.

9. Munier F, Beck-Popovic M, Chantada G, Cobrinik D, Kivelä T, Lohmann D et al. Conservative management of retinoblastoma: Challenging orthodoxy without compromising the state of metastatic grace. "Alive, with good vision and no comorbidity". Progress in Retinal and Eye Research. 2019;73:100764.

10. Azary S, Ganguly A, Bunin GR, Lombardi C, Park AS, Ritz B, et al. Sporadic Retinoblastoma and Parental Smoking and Alcohol Consumption before and after Conception: A Report from the Children's Oncology Group. Plos One. 2016;11(3).

11. Gatta G, Ferrari A, Stiller CA, Pastore G, Bisogno G, Trama A, et al. Embryonal cancers in Europe. European Journal of Cancer. 2012;48(10):1425-33.

12. Andreoli MT, Chau FY, Shapiro MJ, Leiderman YI. Epidemiological trends in 1452 cases of retinoblastoma from the Surveillance, Epidemiology, and End Results (SEER) registry. Canadian Journal of Ophthalmology. 2017;52(6):592-8.

13. Friedrich P, Itriago E, Rodriguez-Galindo C, Ribeiro K. Racial and Ethnic Disparities in the Incidence of Pediatric Extracranial Embryonal Tumors. JNCl: Journal of the National Cancer Institute. 2017;109(10).

14. Francis JH, Roosipu N, Levin AM, Brodie SE, Dunkel IJ, Gobin YP, et al. Current Treatment of Bilateral Retinoblastoma: The Impact of Intraarterial and Intravitreous Chemotherapy. Neoplasia. 2018;20(8):757-63.

15. Knudson AG, Hethcote HW, Brown BW. Mutation and childhood cancer: a probabilistic model for the incidence of retinoblastoma. Proceedings of the National Academy of Sciences. 1975;72(12):511620 . 


\section{CIENCIA\&SALUD}

16. Soliman SE, Racher H, Zhang C, MacDonald H, Gallie BL. Genetics and Molecular Diagnostics in Retinoblastoma-An Update. Asia-Pacific Journal of Ophthalmology. 2017;6(2):197-207.

17. Jr RCE. High-Risk Features and Tumor Differentiation in Retinoblastoma: A Retrospective Histopathologic Study. Archives of Pathology \& Laboratory Medicine. 2009;133(8):1203-9.

18. Lin P, Obrien JM. Frontiers in the Management of Retinoblastoma. American Journal of Ophthalmology. 2009;148(2):192-8.

19. Canturk S, Qaddoumi I, Khetan V, Ma Z, Furmanchuk A, Antoneli CBG, et al. Survival of retinoblastoma in less-developed countries impact of socioeconomic and health-related indicators. British Journal of Ophthalmology. 2010;94(11):1432-6.

20. Meiting Nie. Diagnóstico y tratamiento de retinoblastoma. Rev. costarric. Salud pública [Internet]. 2012 Dec [cited 2021 Oct 03] ; 21( 2 ): 123-126.

21. Butros LJ, Abramson DH, Dunkel IJ. Delayed Diagnosis of Retinoblastoma: Analysis of Degree, Cause, and Potential Consequences. Pediatrics. 2002;109(3).

22. Margo CE, Zimmerman LE. Retinoblastoma: The Accuracy of Clinical Diagnosis in Children Treated by Enucleation. Journal of Pediatric Ophthalmology \& Strabismus. 1983;20(6):227-9

23. Shields $\mathrm{C}$. The International Classification of Retinoblastoma Predicts Chemoreduction Success. Yearbook of Ophthalmology. 2008;2008:230-2.

24. Rodriguez-Galindo C, Orbach DB, Vanderveen D. Retinoblastoma. Pediatric Clinics of North America. 2015;62(1):201-23.

25. Berry J, Jubran R, Wong K, Lee T, Murphree A, Kim J. Factors predictive of long-term visual outcomes of Group D eyes treated with chemoreduction and low-dose IMRT salvage: The Children's Hospital Los Angeles experience. British Journal of Ophthalmology. 2014;98(8):1061-1065.

26. Kivelä T. Trilateral Retinoblastoma: A Meta-Analysis of Hereditary Retinoblastoma Associated With Primary Ectopic Intracranial Retinoblastoma. Journal of Clinical Oncology. 1999;17(6):1829-1829.

27. Kaste S, Jenkins J, Pratt C, Langston J, Haik B. Retinoblastoma. American Journal of Roentgenology. 2000;175(2):495-501.

28. Dhar S. Outcomes of Integrating Genetics in Management of Patients With Retinoblastoma. Archives of Ophthalmology. 2011;129(11):1428.

29. Kim JW, Ngai LK, Sadda S, Murakami Y, Lee DK, Murphree AL. Retcam fluorescein angiography findings in eyes with advanced retinoblastoma. British Journal of Ophthalmology. 2014;98(12):166671.

30. Moulin AP, Gaillard M-C, Balmer A, Munier FL. Ultrasound biomicroscopy evaluation of anterior extension in retinoblastoma: a clinicopathological study. British Journal of Ophthalmology. 2011;96(3):337-40.

31. Kivelä T, Hadjistilianou T. Neonatal retinoblastoma. Asia-Pacific Journal of Oncology Nursing. 2017;4(3):197.

32. Jong MCD, Kors WA, Graaf PD, Castelijns JA, Kivelä T, Moll AC. Trilateral retinoblastoma: a 


\section{CIENCIA\&SALUD}

systematic review and meta-analysis. The Lancet Oncology. 2014;15(10):1157-67.

33. Berry J, Xu L, Murphree A, Krishnan S, Stachelek K, Zolfaghari E et al. Potential of Aqueous Humor as a Surrogate Tumor Biopsy for Retinoblastoma. JAMA Ophthalmology. 2017;135(11):1221.

34. Shields C, Schoenberg E, Kocher K, Shukla S, Kaliki S, Shields J. Lesions Simulating Retinoblastoma (Pseudo-retinoblastoma) in 604 Cases. Ophthalmology. 2013;120(2):311-316.

35. Abramson D, Shields C, Munier F, Chantada G. Treatment of Retinoblastoma in 2015. JAMA Ophthalmology. 2015;133(11):1341.

36. Ortiz M, Dunkel I. (2015). Retinoblastoma. Journal of Child Neurology. 2015;31(2):227-236.

37. Abramson D. Retinoblastoma: Saving Life with Vision. Annual Review of Medicine. 2014;65(1):171184.

38. Dunkel I, Jubran R, Gururangan S, Chantada G, Finlay J, Goldman S et al. Trilateral retinoblastoma: Potentially curable with intensive chemotherapy. Pediatric Blood \& Cancer. 2009;54(3).

39. Jubran R, Erdreich-Epstein A, Butturini A, Murphree A, Villablanca J. Approaches to Treatment for Extraocular Retinoblastoma. Journal of Pediatric Hematology/Oncology. 2004;26(1):31.

40. Abramson D, Daniels A, Marr B, Francis J, Brodie S, Dunkel I et al. Intra-Arterial Chemotherapy (Ophthalmic Artery Chemosurgery) for Group D Retinoblastoma. PLOS ONE. 2016;11(1):e0146582.

41. Jabbour P, Chalouhi N, Tjoumakaris S, Gonzalez L, Dumont A, Chitale R et al. Pearls and pitfalls of intraarterial chemotherapy for retinoblastoma. Journal of Neurosurgery: Pediatrics. 2012;10(3):175181.

42. Shields C, Manjandavida F, Lally S, Pieretti G, Arepalli S, Caywood E et al. Intra-arterial Chemotherapy for Retinoblastoma in 70 Eyes. Ophthalmology.2014;121(7):1453.

43. Yannuzzi N, Francis J, Marr B, Belinsky I, Dunkel I, Gobin Y et al. Enucleation vs Ophthalmic Artery Chemosurgery for Advanced Intraocular Retinoblastoma. JAMA Ophthalmology. 2015;133(9):1062.

44. Wyse E, Handa J, Friedman A, Pearl M. A review of the literature for intra-arterial chemotherapy used to treat retinoblastoma. Pediatric Radiology. 2016;46(9):1223-1233.

45. Kim JW, Kathpalia V, Dunkel IJ, et al Orbital recurrence of retinoblastoma following enucleation British Journal of Ophthalmology 2009;93:463-467.

46. Rodríguez Rodríguez Carlos, Romero Cubero David, Zúñiga Arias Pedro, Picado Rodríguez Luis Alonso. Retinoblastoma: revisión y reporte de un caso. Acta pediátr. costarric [Internet]. 2003 Jan [cited 2021 Oct 03] ; 17( 2): 60-64.

47. Kopelman J, McLean I, Rosenberg S. Multivariate Analysis of Risk Factors for Metastasis in Retinoblastoma Treated by Enucleation. Ophthalmology. 1987;94(4):371-377. 